\title{
Swimming training increases cardiac vagal activity and induces cardiac hypertrophy in rats
}

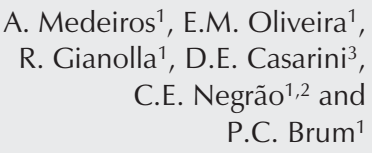

\author{
'Departamento de Biodinâmica do Movimento do Corpo Humano, \\ Escola de Educação Física e Esporte, and ${ }^{2}$ Instituto do Coração, \\ Faculdade de Medicina, Universidade de São Paulo, São Paulo, SP, Brasil \\ ${ }^{3}$ Divisão de Nefrologia, Hospital do Rim e Hipertensão, Escola Paulista de Medicina, \\ Universidade Federal de São Paulo, São Paulo, SP, Brasil
}

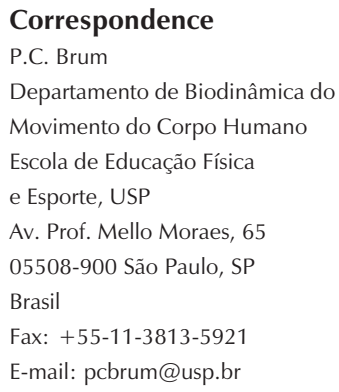

Received October 31, 2003 Accepted August 27, 2004

\begin{abstract}
The effect of swimming training (ST) on vagal and sympathetic cardiac effects was investigated in sedentary $(\mathrm{S}, \mathrm{N}=12)$ and trained $(\mathrm{T}, \mathrm{N}=12)$ male Wistar rats $(200-220 \mathrm{~g})$. ST consisted of 60-min swimming sessions 5 days/week for 8 weeks, with a $5 \%$ body weight load attached to the tail. The effect of the autonomic nervous system in generating training-induced resting bradycardia (RB) was examined indirectly after cardiac muscarinic and adrenergic receptor blockade. Cardiac hypertrophy was evaluated by cardiac weight and myocyte morphometry. Plasma catecholamine concentrations and citrate synthase activity in soleus muscle were also determined in both groups. Resting heart rate was significantly reduced in T rats $(355 \pm 16$ vs 330 $\pm 20 \mathrm{bpm})$. RB was associated with a significantly increased cardiac vagal effect in T rats $(103 \pm 25$ vs $158 \pm 40 \mathrm{bpm})$, since the sympathetic cardiac effect and intrinsic heart rate were similar for the two groups. Likewise, no significant difference was observed for plasma catecholamine concentrations between $\mathrm{S}$ and $\mathrm{T}$ rats. In $\mathrm{T}$ rats, left ventricle weight $(13 \%)$ and myocyte dimension $(21 \%)$ were significantly increased, suggesting cardiac hypertrophy. Skeletal muscle citrate synthase activity was significantly increased by $52 \%$ in $\mathrm{T}$ rats, indicating endurance conditioning. These data suggest that RB induced by ST is mainly mediated parasympathetically and differs from other training modes, like running, that seems to mainly decrease intrinsic heart rate in rats. The increased cardiac vagal activity associated with ST is of clinical relevance, since both are related to increased life expectancy and prevention of cardiac events.
\end{abstract}

\section{Introduction}

Exercise training has been associated with several metabolic, cardiovascular and neurovegetative benefits. Resting bradycardia has been considered to be the hallmark cardiovascular effect of exercise-training adaptation in both animals $(1,2)$ and humans $(3)$.
Key words

- Swimming-training exercise

- Cardiac autonomic balance

- Hemodynamics

- Hypertrophic effect

- Parasympathetic system

- Resting bradycardia 
investigating the sympatho-vagal balance by spectral analysis have shown increased cardiac vagal tone in exercise-trained humans (8). Alternatively, reduction in intrinsic heart rate (IHR) in exercise-trained rats (9) and exercise-trained humans (10), and more recently longer atrio-ventricular conduction time (11) have led some investigators to attribute resting bradycardia to intrinsic cardiac alteration. These contrasting results might be due to the methodology used to analyze the cardiac autonomic balance, the species studied, and probably to the different training regimens and modes applied.

Cardiac autonomic balance after running training has been extensively studied $(5,9,12)$. In contrast, little is known about swimming as a training mode. Swimming is well recognized for its effectiveness in inducing myocardial hypertrophy $(2,13)$ and left ventricular (LV) end-diastolic volume increase in rats (14). In addition, swimming seems to produce a greater resting bradycardia than running (15). However, the mechanisms involved in swimming-training bradycardia are not completely understood. Therefore, the purpose of the present investigation was to study the vagal and sympathetic control of heart rate (HR) in swimming-trained rats. We report here that swimming increased the cardiac vagal effect in rats, but caused no change in either cardiac sympathetic effect or plasma catecholamine concentration. These rats also presented cardiac hypertrophy. These results support the view of the specificity of cardiac adaptation to endurance training, and strongly suggest that swimming-training bradycardia in rats resulted from an increased cardiac vagal effect.

\section{Material and Methods}

\section{Animals care and swimming-training protocol}

Twenty-four normotensive male Wistar rats (200 to $220 \mathrm{~g}$ ) were housed 3-5 per cage in a temperature-controlled room $\left(22^{\circ} \mathrm{C}\right)$ with a 12-h dark-light cycle, with free access to standard laboratory chow and tap water. The study was conducted according to the ethical principles in animal research adopted by the Brazilian College of Animal Experimentation.

Rats were randomly assigned to sedentary $(\mathrm{N}=12)$ and swimming-trained $(\mathrm{N}=12)$ groups. The training sessions were performed during the rats' dark cycle and consisted of five day/week swimming sessions of 60-min duration for 8 weeks in an apparatus adapted for rats containing warmed water $\left(30-32^{\circ} \mathrm{C}\right)$. Exercise duration and workload were increased gradually until the rats could swim for 60 min wearing caudal dumbbells weighing $5 \%$ of their body weight. Thereafter, duration and workload were constant. Sedentary rats were placed in the swimming apparatus for 10 min twice a week to mimic the water stress associated with the experimental protocol. This swimming protocol has been characterized previously as low to moderate intensity and long duration due to improvement in muscle oxidative capacity (16).

All the rats were weighed weekly, and tail-cuff systolic blood pressure (BP) and HR were determined twice a week throughout the experimental period.

\section{Tail-cuff blood pressure measurements}

$\mathrm{BP}$ and HR were measured during the nine-week study period using a computerized tail-cuff system (Kent Scientific, Torrington, CT, USA). For BP measurement, rats were placed in a warmed rat restraining apparatus during their dark cycle. A cuff was placed around the rat tail and insufflated until blood flow was occluded, and then released until the first pulses of arterial flow could be detected and recorded on a microcomputer (AT/CODAS, 100-Hz sampling rate, DataQ Instruments, Inc., Akron, $\mathrm{OH}$, USA). Six BP measurements were performed 
twice a week, but only 3 technically successful ones were taken into consideration. Next, $\mathrm{BP}$ was determined for each animal by averaging the values obtained during the week. HR was obtained from BP pulse records.

\section{Cardiac autonomic control}

We evaluated the resting HR $24 \mathrm{~h}$ after the last swimming-training session as a measure of cardiac autonomic control. Three stainless steel electrodes $(5 \mathrm{~cm}$ long and $0.55 \mathrm{~mm}$ in diameter) were implanted subcutaneously under ketamine $(50 \mathrm{mg} / \mathrm{kg}$, ip $)$ and xylazine $(10 \mathrm{mg} / \mathrm{kg}$, ip) anesthesia, and attached to the right and left axillary areas and dorsum $(17,18)$. The electrodes were used to monitor the electrocardiographic (EKG) results with a multichannel recorder (model 7754A; Hewlett-Packard, Palo Alto, CA, USA) as measures of HR. Simultaneously, the EKG signal was fed to a 16-channel analog-todigital converter and then to a microcomputer using AT/CODAS at $500 \mathrm{~Hz}$ (DataQ Instruments).

Vagal and sympathetic effects were studied during the rats' dark cycle by intravenous injections of $3 \mathrm{mg} / \mathrm{kg}$ methylatropine and 4 $\mathrm{mg} / \mathrm{kg}$ propranolol (Sigma, St. Louis, MO, USA), $24 \mathrm{~h}$ after electrode implantation. On the first day of the study, the resting HR was recorded in quiet unrestrained rats kept in their individual cages and methylatropine was injected immediately after recording the resting HR. Because the HR response to methylatropine reaches its peak in 10-15 min, this interval was standardized before the HR measurement. Propranolol was injected 15 min after methylatropine, and HR was measured again after 10-15 min.

The IHR was evaluated after simultaneous blockade with propranolol and methylatropine. We observed that these drugs were still promoting a blockade $30 \mathrm{~min}$ after injection. On the 2nd day of the study, propranolol was administered first to obtain the inverse sequence of blockade.
The vagal effect was evaluated as the difference between the maximum HR after methylatropine injection and the control HR. The sympathetic effect was evaluated as the difference between the control HR and minimum HR after propranolol injection. The vagal and sympathetic effect terminology has been used and published previously by our laboratory (12), and is used to estimate the vagal and sympathetic contribution to the maintenance of control HR.

At the end of the experiment, the animals were weighed and killed by decapitation. The heart was rapidly excised and immersed in a $1 \mathrm{M} \mathrm{KCl}$-based solution in order to be arrested at diastole. Ventricular tissues were dissected and processed immediately and skeletal muscle (soleus) was frozen at $-80^{\circ} \mathrm{C}$ until analysis.

\section{Plasma catecholamine concentrations}

Catecholamines (norepinephrine, epinephrine and dopamine) were measured by HPLC using ion-pair reverse phase chromatography coupled with electrochemical detection $(0.5 \mathrm{~V})$ as described by NaffahMazzacoratti et al. (19). Isocratic separation was obtained using an RP 18 Brownlee column $(4.6$ x $250 \mathrm{~mm}, 5 \mu$; Applied Biosystems, San Jose, CA, USA) eluted with the following mobile phase: $20 \mathrm{mM}$ sodium dibasic phosphate, $20 \mathrm{mM}$ citric acid, $\mathrm{pH} 2.64$, containing $10 \%$ methanol, $0.12 \mathrm{mM} \mathrm{Na}$ EDTA, and $566 \mathrm{mg} / \mathrm{l}$ heptanesulfonic acid. The total time for sample analysis was 30 $\mathrm{min}$. The quantitation of catecholamines was achieved by comparing peak areas of samples with those of standards and using dihydroxybenzylamine (DHBA) as an internal standard. Plasma $(0.5 \mathrm{ml})$ was added to $50 \mathrm{mg}$ $\mathrm{Al}_{2} \mathrm{O}_{5}$ in a centrifuge tube and $3.5 \mathrm{ml} 370$ mM Tris-buffer, $\mathrm{pH} 8.8$, plus $40 \mu \mathrm{l}$ (8 ng) of DHBA as the internal standard were added. The suspension was vortex-mixed for 10 min and the alumina was washed three times with $1 \mathrm{ml}$ water by vortex mixing. The cat- 
echolamines were eluted with $400 \mu \mathrm{l} 100 \mathrm{mM}$ perchloric acid after 3 min of vortex mixing. After centrifugation for $3 \mathrm{~min}$ at $2,500 \mathrm{~g}$, the supernatant was filtered and $100 \mu \mathrm{l}$ was injected into the reverse phase column.

\section{Analysis of cardiac structure}

After removal from the thoracic cavity the heart was trimmed of excess non-cardiac tissues and dissected to obtain the LV (free wall plus septum) weight. Hypertrophy is reported as increase in LV weight normalized for body weight (LV weight/BW).

LV myocardium obtained from the free wall at the level of the papillary muscle was fixed in $6 \%$ formaldehyde and embedded in paraffin, cut into 5- $\mu \mathrm{m}$ sections and subsequently stained with hematoxylin and eosin. Two sections were randomly selected for each animal. All sections were examined by light microscopy using an oil immersion objective with a fixed magnification of 400X. Myocytes were selected for diameter determination if a visible nucleus was present and their cell membranes were intact. The cardiomyocyte image was displayed on a viewing screen, and the widths of each individually isolated cardiac myocyte were manually

Figure 1. Systolic blood pressure (SBP, Panel A) and heart rate $(\mathrm{HR}$, Panel B) during the weeks of exercise training or inactivity. 0 , Before exercise training or inactivity period. Data are reported as means \pm SEM for 12 rats in each group. Note that SBP values were similar for sedentary (S) and swimming-trained (T) rats, while a significant resting bradycardia was observed in $\mathrm{T}$ rats following the sixth week of exercise training. ${ }^{*} \mathrm{P} \leq 0.05 \mathrm{com}$ pared to $T$ rats (one-way ANOVA for repeated measures followed by post hoc Tukey test).

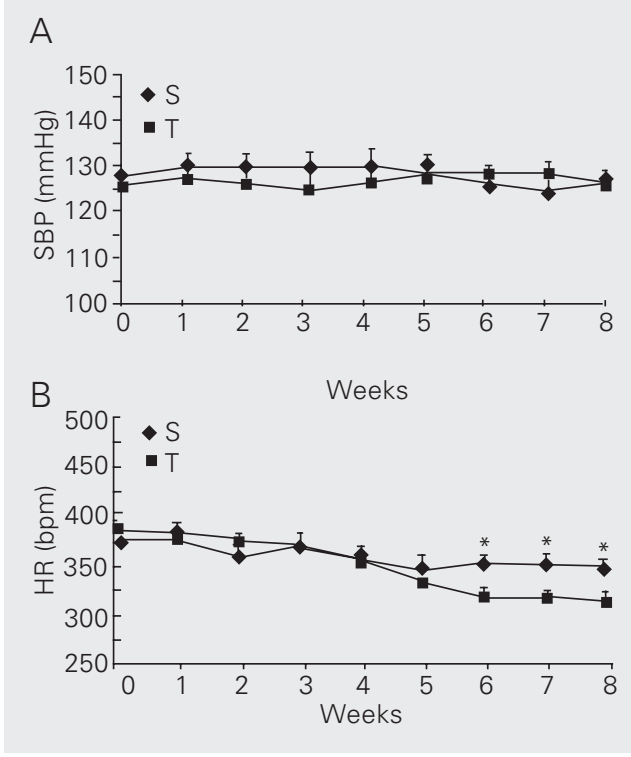

traced across the middle of the nucleus with a digitizing pad. A computer-assisted image analysis was used to calculate the traced area (Quantimet 520; Cambridge Instruments, Woburn, MA, USA). Myocyte diameters were measured in approximately 20 visual fields.

\section{Skeletal muscle oxidative enzyme activity}

Soleus muscle citrate synthase activity, a marker of the tricarboxylic acid cycle, was measured spectrophotometrically. Aliquots of muscle samples were homogenized 1:10 in PBS for enzymatic analysis. The assay of citrate synthase, whose enzyme activity was determined following the production of a yellow compound which results from the reaction between DTNB and the CoA released, consisted of $100 \mathrm{mmol}$ Tris- $\mathrm{HCl} / \mathrm{l}$, 0.2 mmol 5,5'-dithio-bis-2-nitrobenzoic acid, $15 \mathrm{mmol}$ acetyl-CoA/l, and $0.5 \mathrm{mmol}$ oxaloacetate/l, $\mathrm{pH} 8.1$. Citrate synthase activity is reported as $\mu \mathrm{mol} \mathrm{min}^{-1} \mathrm{mg}$ protein ${ }^{-1}$.

\section{Statistical analysis}

Data are reported as means \pm SD. Tailcuff BP and HR data were compared between groups by one-way ANOVA for repeated measures followed by the post hoc Tukey test. For autonomic and structural cardiac data, plasma catecholamines, and muscle oxidative capacity, the unpaired Student $t$-test was used to compare means. The level of significance was set at $\mathrm{P} \leq 0.05$ for all analyses.

\section{Results} Resting systolic blood pressure and heart
rate during the swimming-training period

Baseline systolic BP did not differ between groups throughout the eight weeks of the experimental protocol (Figure 1A). However, HR decreased significantly after 6 
weeks of swimming training compared with the pre-exercising period and/or sedentary littermates (Figure 1B). In contrast, HR was unchanged in the sedentary group throughout the study. The development of resting bradycardia in the swimming-trained group indicates that aerobic conditioning was achieved under the present conditions. Moreover, the presence of swimming-training bradycardia was confirmed by EKG studies (Figure 2A).

\section{Cardiac autonomic control}

To study the mechanism involved in resting bradycardia after swimming training, the IHR and sympathetic and parasympathetic cardiac autonomic controls were calculated. IHR, measured after simultaneous autonomic blockade obtained after propranolol and methylatropine administration, was not different between groups (Figure 2B).

The sympathetic effect did not differ between sedentary and swimming-trained groups (Figure 2C), whereas the vagal effect increased significantly in swimming-trained rats compared with sedentary rats (Figure 2D). These data indicate that swimmingtraining bradycardia is probably due to an increased cardiac vagal effect.

\section{Plasma catecholamine concentrations}

To further investigate sympathetic activity after swimming training we measured plasma catecholamine concentrations. There was no difference in plasma norepinephrine, epinephrine, L-dopamine, and dopamine concentrations $(5365 \pm 776$ vs $6439 \pm 824$, $6154 \pm 665$ vs $7753 \pm 841,200 \pm 25$ vs $156 \pm$ 20 , and $90 \pm 25$ vs $102 \pm 20 \mathrm{pg} / \mathrm{mg}$ protein, respectively) between sedentary and swimming-trained rats.

\section{Cardiac structure analysis}

The BW of swimming-trained rats were similar to those of sedentary rats (Table 1). However, cardiac hypertrophy was observed in the swimming-trained group. LV/BW was significantly higher (13\%) in the swimmingtrained group. Likewise, both right ventricle (18\%) and total heart weight (13\%) were significantly increased in swimming-trained rats. The change in LV weight was further confirmed by a $21 \%$ significant increase in LV myocyte diameter when swimming-trained rats were compared to sedentary rats (Table 1).

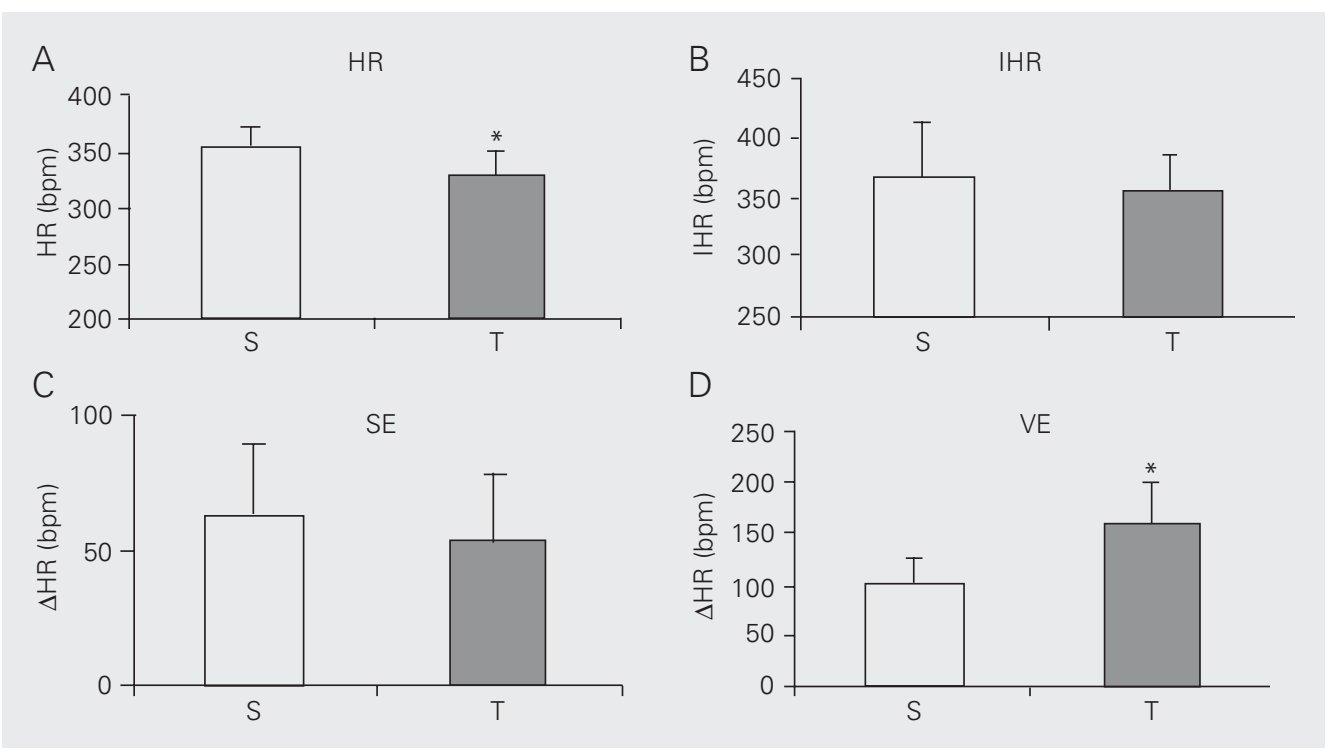

Figure 2. Cardiac autonomic control of heart rate in sedentary (S) and swimming-trained (T) rats. A, Resting heart rate $(\mathrm{HR}) ; B$, intrinsic heart rate $(\mathrm{IHR})$; $C$, cardiac sympathetic effect (SE); $D$, cardiac vagal effect (VE). Data are reported as means \pm SEM for 12 rats in each group. Note that the resting bradycardia observed in $T$ rats was attributed to increased VE since SE and IHR values were similar for $S$ and $T$ rats. ${ }^{*} P \leq 0.05 \mathrm{com}$ pared to $S$ rats (unpaired Student $t$-test). 


\section{Skeletal muscle oxidative enzyme activity}

The soleus muscle has been used for studies on slow-twitch red fiber oxidative capacity (20). Citrate synthase, a respiratory enzyme which has been shown (20) to undergo adaptive increases due to exercise in skeletal muscle fibers, was also used as a training marker in the present study. We observed a $52 \%$ increase in citrate synthase activity of soleus muscle in swimming-trained rats when compared with sedentary rats $\left(157.8 \pm 2.2\right.$ vs $\left.103.8 \pm 2.6 \mu \mathrm{mol} \mathrm{mg}^{-1} \mathrm{ml}^{-1}\right)$.

\section{Discussion}

The main findings of the present investigation were that: 1) an 8-week swimmingtraining period significantly decreases resting HR in rats; 2) swimming training-induced bradycardia was associated with an increased cardiac vagal effect, but not with a sympathetic cardiac effect or IHR, and 3) swimming training induced cardiac hypertrophy in rats.

\section{Heart rate adaptations}

The present findings show that the mechanisms underlying the resting bradycardia are strongly dependent on exercise training mode. In contrast to previous studies, in which resting bradycardia after running training was explained by a reduction in IHR $(12,21)$ or reduced atrioventricular con- duction (11) in man and rats, we provide evidence here for vagal-mediated resting bradycardia after swimming training in rats. In fact, a different cardiac adaptation after swimming training should be expected, since this training mode differs from running training with respect to body position in the water, water pressure and temperature regulation. Indeed, few studies comparing the effect of different endurance training modes on the autonomic nervous system have been conducted on other species. In humans, cardiac vagal tone analyzed by spectral analysis did not differ between runners and swimmers (22). In contrast, in running-trained mice, the resting bradycardia was associated with an increased cardiac vagal tone concomitantly with a decreased sympathetic tone (1), while in swimming-trained mice it was associated with a decreased cardiac sympathetic tone (Medeiros A and Brum PC, unpublished data).

The resting HR adaptation observed here cannot be attributed to sympathetic attenuation since both cardiac sympathetic effect and plasma catecholamine levels were unchanged in swimming-trained rats. Whether similar swimming adaptations take place in humans is still under investigation. Cardiac adaptations such as vagal predominance and a mild increase in LV dimensions are already observed in prepubertal swimmers (23). Adult swimming athletes examined during their yearly rest presented resting bradycardia associated with an increased vagal tone

Table 1. Gross cardiac region weights and myocyte diameter of sedentary (S) and swimming-trained (T) rats.

\begin{tabular}{lccccccc}
\hline & $N$ & $\begin{array}{c}\text { BW } \\
(\mathrm{g})\end{array}$ & $\begin{array}{c}\text { AT W/BW } \\
(\mathrm{mg} / \mathrm{g})\end{array}$ & $\begin{array}{c}\text { RV W/BW } \\
(\mathrm{mg} / \mathrm{g})\end{array}$ & $\begin{array}{c}\text { LV W/BW } \\
(\mathrm{mg} / \mathrm{g})\end{array}$ & $\begin{array}{c}\mathrm{H} \text { W/BW } \\
(\mathrm{mg} / \mathrm{g})\end{array}$ & $\begin{array}{c}\text { LV myocyte } \\
\text { diameter }(\mu \mathrm{m})\end{array}$ \\
\hline $\mathrm{S}$ & 12 & $360 \pm 27$ & $0.15 \pm 0.02$ & $0.56 \pm 0.04$ & $1.95 \pm 0.14$ & $2.82 \pm 0.16$ & $10.0 \pm 0.02$ \\
$\mathrm{~T}$ & 12 & $361 \pm 15$ & $0.16 \pm 0.02$ & $0.66 \pm 0.05^{*}$ & $2.20 \pm 0.15^{*}$ & $3.18 \pm 0.21^{*}$ & $12.0 \pm 0.03^{*}$ \\
$\%$ Change & - & - & 8 & 18 & 13 & 13 & 21
\end{tabular}

Data are reported as means \pm SEM for 12 rats in each group. $B W=$ body weight; $A T$ T $/ B W=$ atrium weight to body weight ratio; RV W/BW = right ventricle weight to body weight ratio; $\mathrm{LV} W / \mathrm{BW}=$ left ventricle weight to body weight ratio; $\mathrm{H}$ W/BW = heart weight to body weight ratio; LV, left ventricle.

${ }^{*} \mathrm{P} \leq 0.05$ compared to $S$ rats (unpaired Student $t$-test). 
(24). However, champion swimmers examined at the peak of their training season showed a resting bradycardia paradoxically coexisting with an increased cardiac sympathetic tone (24). Recently, similar results were observed for highly fit rowers, who showed a shift in cardiovascular autonomic modulation from parasympathetic to sympathetic predominance during the World Championship (25). Therefore, the cardiac autonomic control seems to be influenced by either training mode or season, a fact that represents an interesting topic for future investigations. Indeed, recent studies have demonstrated that vagal improvement is associated with lower adverse cardiac events such as, lower cardiac arrhythmias $(9,26)$. Conversely, cardiac sympathetic predominance may be hazardous to the cardiovascular system since it triggers arrhythmias $(27,28)$.

Another interesting finding of the present study regards the time needed for HR reduction. Resting bradycardia did not occur before six weeks of swimming training. This raises the possibility of a long-term effect of exercise training, especially because 6 weeks is a long time for a rat.

\section{Cardiac adaptations}

Earlier studies have also demonstrated that exercise training by swimming increases the heart weight to BW ratio to values ranging from 12 to $31 \%$ in rats (29). Cardiac hypertrophy is known to occur in response to various stimuli such as pressure and volume overload. Also, the association between LV structure at rest and cardiac performance during physical effort has been emphasized by several studies performed on trained subjects $(30,31)$. Endurance-trained subjects involved in training with a high dynamic aerobic component develop predominantly increased LV chamber size, with a proportional increase in wall thickness and LV stroke volume caused by volume overload. Thus, they show eccentric cardiac hypertro- phy associated with improved cardiac performance during exercise. On the other hand, strength-trained subjects, mainly involved in static isometric anaerobic exercise, show increased LV wall thickness with a pattern of concentric geometry caused by pressure overload during exercise.

Swimming training, used here as a training mode, is mainly related to volume overload-induced cardiac eccentric hypertrophy with predominant longitudinal myocyte growth (32). Although myocyte length was not determined in the present study, we were able to detect an increase in myocyte width which paralleled the changes observed in LV/BW ratios. This is consistent with the idea that exercise training-induced eccentric hypertrophy can lead to a proportional myocyte width and length growth (33). Moreover, the cardiac hypertrophy observed in swimming-trained rats is a physiological and beneficial cardiac adaptation, usually associated with increased cardiac performance.

\section{Skeletal muscle adaptations}

The increase in skeletal muscle oxidative capacity by exercise training is a well-established adaptation (34) and is considered a good marker for exercise training efficiency (35). In our study, we found a $52 \%$ increase in citrate synthase activity in swimmingtrained rats when compared with sedentary rats. These results show the effectiveness of the swimming-training protocol, and should be taken into consideration in future investigations dealing with swimming-training protocols in rats.

\section{Blood pressure adaptations}

The lack of exercise-training adaptation in systolic BP in normotensive rats is consistent with previous observations in studies by our group $(36,37)$ and others (38). In fact, exercise training has been recommended as a non-pharmacological treatment for high 
blood pressure, but its effect in normotensive animals and normotensive humans seems to be minimal.

We have extended previous observations of the effect of training on the cardiovascular system by showing that the resting bradycardia occurs as a consequence of an increased cardiac vagal effect in swimming-trained rats. Furthermore, we demonstrated that swimming training is efficient in inducing cardiac hypertrophy in rats. This demonstrates the importance of considering the nature of the training mode and species in cardiac autonomic and structural adaptations. Furthermore, these alterations, if reproducible in humans, are of clinical relevance since both a reduction in HR and an increase in vagal tone have been associated with an increase in life expectancy (39) and a protective effect against cardiac events such as lethal arrhythmias (40).

\section{Acknowledgments}

The authors thank Katt Coelho Mattos (Departamento de Biodinâmica do Movimento do Corpo Humano, Escola de Educação Física e Esporte, USP, São Paulo, SP, Brazil) for technical assistance.

\section{References}

1. De Angelis K, Wichi RB, Jesus WR, Moreira ED, Morris M \& Krieger EM (2004). Exercise training changes autonomic cardiovascular balance in mice. Journal of Applied Physiology, 96: 2174-2178.

2. Evangelista FS, Brum PC \& Krieger JE (2003). Duration-controlled swimming exercise training induces cardiac hypertrophy in mice. Brazilian Journal of Medical and Biological Research, 36: 17511759.

3. Katona PG, McLean M, Dighton DH \& Guz A (1982). Sympathetic and parasympathetic cardiac control in athletes and nonathletes at rest. Journal of Applied Physiology, 52: 1652-1657.

4. Wisloff U, Loennechen JP, Falck G, Beisvag V, Currie S, Smith G \& Ellingsen $O$ (2001). Increased contractility and calcium sensitivity in cardiac myocytes isolated from endurance trained rats. Cardiovascular Research, 50: 495-508.

5. Shi X, Stevens GH, Foresman BH, Stern SA \& Raven PB (1995). Autonomic nervous system control of the heart: endurance exercise training. Medicine and Science in Sports and Exercise, 27: 1406-1413.

6. Jost J, Weiss M \& Weicker H (1989). Comparison of sympathoadrenergic regulation at rest and of the adrenoceptor system in swimmers, long-distance runners, weight lifters, wrestlers and untrained men. European Journal of Applied Physiology and Occupational Physiology, 58: 596-604.

7. Lin YC \& Horvath SM (1972). Autonomic nervous control of cardiac frequency in the exercise-trained rat. Journal of Applied Physiology, 33: 796-799

8. Yamamoto K, Miyachi M, Saitoh T, Yoshioka A \& Onodera S (2001). Effects of endurance training on resting and post-exercise cardiac autonomic control. Medicine and Science in Sports Exercise, 33: 1496-1502.

9. Bolter CP, Hughson RL \& Critz JB (1973). Intrinsic rate and cholinergic sensitivity of isolated atria from trained and sedentary rats. Proceedings of the Society for Experimental Biology and Medicine, 144: 364-367.

10. Catai AM, Chacon-Mikahil MP, Martinelli FS et al. (2002). Effects of aerobic exercise training on heart rate variability during wakeful- ness and sleep and cardiorespiratory responses of young and middle-aged healthy men. Brazilian Journal of Medical and Biological Research, 35: 741-752.

11. Stein R, Medeiros CM, Rosito GA, Zimerman LI \& Ribeiro JP (2002). Intrinsic sinus and atrioventricular node electrophysiologic adaptations in endurance athletes. Journal of the American College of Cardiology, 39: 1033-1038.

12. Negrao CE, Moreira ED, Santos MC, Farah VM \& Krieger EM (1992). Vagal function impairment after exercise training. Journal of Applied Physiology, 72: 1749-1753.

13. Kaplan ML, Cheslow Y, Vikstrom K, Malhotra A, Geenen DL, Nakouz A, Leinwand LA \& Buttrick PM (1994). Cardiac adaptations to chronic exercise in mice. American Journal of Physiology, 267: H1167H1173.

14. Geenen D, Buttrick P \& Scheuer J (1988). Cardiovascular and hormonal responses to swimming and running in the rat. Journal of Applied Physiology, 65: 116-123.

15. Schaible TF \& Scheuer J (1979). Effects of physical training by running or swimming on ventricular performance of rat hearts. Journal of Applied Physiology, 46: 854-860.

16. Medeiros A, Gianolla RM, Kalil LMP, Bacurau RFP, Rosa LFBC, Negrao CE \& Brum PC (2000). Efeito do treinamento físico de natação sobre o sistema cardiovascular de ratos normotensos. Revista Paulista de Educação Física, 14: 7-15.

17. Machado $B H$, Krieger EM \& Salgado HC (1987). Changes of the intrinsic heart rate during the onset of renal hypertension. Journal of Hypertension, 5: 755-759.

18. Soato GG \& Krieger EM (1974). Heart rate after acute hypertension in the rat. American Journal of Physiology, 227: 1389-1393.

19. Naffah-Mazzacoratti MC, Casarini DE, Fernandes MJS \& Cavalheiro EA (1992). Serum catecholamine levels determined by high performance liquid chromatography coupled with electrochemical detection. Arquivos Brasileiros de Endocrinologia e Metabolismo, 36: 119-122.

20. Baldwin KM, Klinkerfuss GH, Terjung RL, Mole PA \& Holloszy JO (1972). Respiratory capacity of white, red, and intermediate muscle: 
adaptative response to exercise. American Journal of Physiology, 222: 373-378.

21. Schaefer ME, Allert JA, Adams HR \& Laughlin MH (1992). Adrenergic responsiveness and intrinsic sinoatrial automaticity of exercisetrained rats. Medicine and Science in Sports and Exercise, 24: 887894.

22. Franke WD, Mills KK, Lee K \& Hernandez JP (2003). Training mode does not affect orthostatic tolerance in chronically exercising subjects. European Journal of Applied Physiology, 89: 263-270.

23. Triposkiadis F, Ghiokas S, Skoularigis I, Kotsakis A, Giannakoulis I \& Thanopoulos $V$ (2002). Cardiac adaptation to intensive training in prepubertal swimmers. European Journal of Clinical Investigation, 32: 16-23.

24. Furlan R, Pagani M \& Malliani A (1993). Effects of exercise and training on control of heart rate. Cardiovascular Research, 27: 22862287.

25. Iellamo F, Legramante JM, Pigozzi F, Spataro A, Norbiato G, Lucini $D$ \& Pagani M (2002). Conversion from vagal to sympathetic predominance with strenuous training in high-performance world class athletes. Circulation, 105: 2719-2724.

26. Roveda F, Middlekauff HR, Rondon MU, Reis SF, Souza M, Nastari L, Barreto AC, Krieger EM \& Negrao CE (2003). The effects of exercise training on sympathetic neural activation in advanced heart failure: a randomized controlled trial. Journal of the American College of Cardiology, 42: 854-860.

27. Stein KM, Karagounis LA, Anderson JL, Kligfield $P$ \& Lerman BB (1995). Fractal clustering of ventricular ectopy correlates with sympathetic tone preceding ectopic beats. Circulation, 91: 722-727.

28. Adamopoulos S, Parissis JT \& Kremastinos DT (2003). New aspects for the role of physical training in the management of patients with chronic heart failure. International Journal of Cardiology, 90: 1-14.

29. Schaible TF \& Scheuer J (1981). Cardiac function in hypertrophied hearts from chronically exercised female rats. Journal of Applied Physiology, 50: 1140-1145.

30. D'Andrea A, Limongelli G, Caso P, Sarubbi B, Della Pietra A, Brancaccio P, Cice G, Scherillo M, Limongelli F \& Calabro R (2002).
Association between left ventricular structure and cardiac performance during effort in two morphological forms of athlete's heart. International Journal of Cardiology, 86: 177-184.

31. Pluim BM, Zwinderman $A H$, van der Laarse $A$ \& van der Wall EE (2000). The athlete's heart. A meta-analysis of cardiac structure and function. Circulation, 101: 336-344.

32. Lorell BH \& Carabello BA (2000). Left ventricular hypertrophy: pathogenesis, detection, and prognosis. Circulation, 102: 470-479.

33. Claessens C, Claessens P, Bloemen H, Claessens M, Verbanck M, Fagard R \& Claessens J (1999). Structural heart adaptations in triathletes. Acta Cardiologica, 54: 317-325.

34. Srere PA (1968). Studies on purified citrate-enzymes: metabolic interpretations. Biochemical Society Symposia, 27: 11-21.

35. Wibom R, Hultman E, Johansson M, Matherei K, ConstantinTeodosiu D \& Schantz PG (1992). Adaptation of mitochondrial ATP production in human skeletal muscle to endurance training and detraining. Journal of Applied Physiology, 73: 2004-2010.

36. Negrao CE, Irigoyen MC, Moreira ED, Brum PC, Freire PM \& Krieger EM (1993). Effect of exercise training on RSNA, baroreflex control, and blood pressure responsiveness. American Journal of Physiology, 265: R365-R370.

37. Krieger EM, Brum PC \& Negrao CE (1998). Role of arterial baroreceptor function on cardiovascular adjustments to acute and chronic dynamic exercise. Biological Research, 31: 273-279.

38. Desai KH, Sato R, Schauble E, Barsh GS, Kobilka BK \& Bernstein D (1997). Cardiovascular indexes in the mouse at rest and with exercise: new tools to study models of cardiac disease. American Journal of Physiology, 272: H1053-H1061.

39. Paffenbarger Jr RS, Blair SN, Lee IM \& Hyde RT (1993). Measurement of physical activity to assess health effects in free-living populations. Medicine and Science in Sports and Exercise, 25: 6070.

40. Berlin JA \& Colditz GA (1990). A meta-analysis of physical activity in the prevention of coronary heart disease. American Journal of Epidemiology, 132: 612-628. 